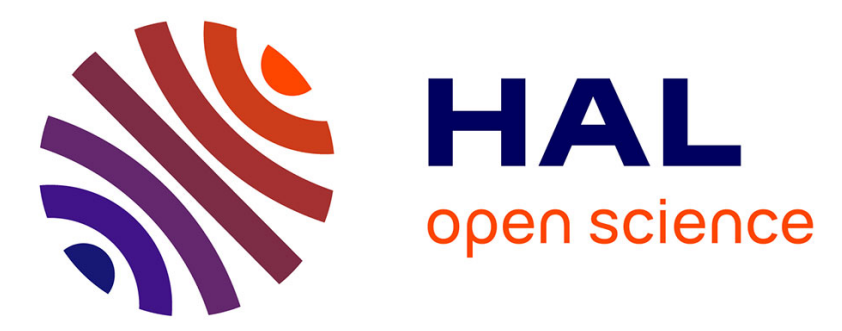

\title{
Kinetic Interaction of Cold and Hot Protons With an Oblique EMIC Wave Near the Dayside Reconnecting Magnetopause
}

S. Toledo-redondo, J. Lee, S. Vines, D. Turner, R. Allen, M. André, S. Boardsen, J. Burch, R. Denton, H. Fu, et al.

\section{To cite this version:}

S. Toledo-redondo, J. Lee, S. Vines, D. Turner, R. Allen, et al.. Kinetic Interaction of Cold and Hot Protons With an Oblique EMIC Wave Near the Dayside Reconnecting Magnetopause. Geophysical Research Letters, 2021, 48 (8), 10.1029/2021GL092376 . hal-03329910

\section{HAL Id: hal-03329910 https://hal.science/hal-03329910}

Submitted on 2 Sep 2021

HAL is a multi-disciplinary open access archive for the deposit and dissemination of scientific research documents, whether they are published or not. The documents may come from teaching and research institutions in France or abroad, or from public or private research centers.
L'archive ouverte pluridisciplinaire HAL, est destinée au dépôt et à la diffusion de documents scientifiques de niveau recherche, publiés ou non, émanant des établissements d'enseignement et de recherche français ou étrangers, des laboratoires publics ou privés. 


\section{Geophysical Research Letters}

\section{RESEARCH LETTER \\ 10.1029/2021GL092376 \\ Special Section: \\ Probing the Magnetosphere through Magnetoseismology and Ultra-Low-Frequency \\ Waves \\ Key Points: \\ - In situ observation of different dynamics of cold (eV) and hot (keV) protons inside an electromagnetic ion cyclotron wave \\ - Wave number estimation shows that cold protons behave as fluid while hot protons interact at kinetic scales \\ - Magnetized cold protons modify the Ohm's law balance and favor propagation at a large wave normal angle}

Supporting Information:

Supporting Information may be found in the online version of this article.

Correspondence to:

S. Toledo-Redondo,

sergio.toledo@um.es

Citation:

Toledo-Redondo, S., Lee, J. H., Vines, S. K., Turner, D. L., Allen, R. C., André, M., et al. (2021). Kinetic interaction of cold and hot protons with an oblique EMIC wave near the dayside reconnecting magnetopause. Geophysical Research Letters, 48, e2021GL092376. https://doi. org/10.1029/2021GL092376

Received 4 JAN 2021 Accepted 8 APR 2021
(C) 2021. The Authors.

This is an open access article under the terms of the Creative Commons Attribution License, which permits use, distribution and reproduction in any medium, provided the original work is properly cited.

\section{Kinetic Interaction of Cold and Hot Protons With an Oblique EMIC Wave Near the Dayside Reconnecting Magnetopause}

\author{
S. Toledo-Redondo ${ }^{1,2}$ (D) J. H. Lee ${ }^{3}$ (D) S. K. Vines ${ }^{4}$ (D), D. L. Turner ${ }^{4}$ (D) R. C. Allen ${ }^{4}$ (D), \\ M. André ${ }^{5}$ (D), S. A. Boardsen ${ }^{6}$ (D), J. L. Burch ${ }^{7}$ (D) R. E. Denton ${ }^{8}$ (D), H. S. Fu' (D),

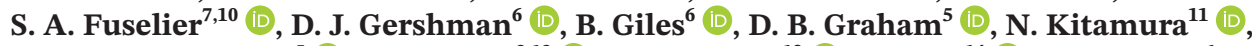 \\ Yu. V. Khotyaintsev ${ }^{5}$ (D) B. Lavraud ${ }^{2,12}$ (D) O. Le Contel ${ }^{13}$ (D) W. Y. Li ${ }^{14}$ (D) T. E. Moore ${ }^{6}$, \\ E. A. Navarro ${ }^{15}$ (D) J. Portí ${ }^{16}$ (D) A. Salinas ${ }^{17}$ (iD) and A. Vinas ${ }^{6}$ (D)
}

\begin{abstract}
${ }^{1}$ Department of Electromagnetism and Electronics, University of Murcia, Murcia, Spain, ${ }^{2}$ Institut de Recherche en Astrophysique et Planétologie, Université de Toulouse, CNRS, UPS, CNES, Toulouse, France, ${ }^{3}$ The Aerospace Corporation, El Segundo, CA, USA, ${ }^{4}$ The Johns Hopkins University Applied Physics Laboratory, Laurel, MD, USA, ${ }^{5}$ Swedish Institute of Space Physics, Uppsala, Sweden, ${ }^{6}$ NASA Goddard Space Flight Center, Greenbelt, MD, USA, ${ }^{7}$ Southwest Research Institute, San Antonio, TX, USA, ${ }^{8}$ Department of Physics and Astronomy, Dartmouth College, Hanover, NH, USA, ${ }^{9}$ School of Space and Environment, Beihang University, Beijing, China, ${ }^{10}$ University of Texas at San Antonio, San Antonio, TX, USA, ${ }^{11}$ Department of Earth and Planetary Science, Graduate School of Science, University of Tokyo, Tokyo, Japan, ${ }^{12}$ Laboratoire d'Astrophysique de Bordeaux, Université de Bordeaux, CNRS, Pessac, France, ${ }^{13}$ Laboratoire de Physique des Plasmas (LPP), UMR7648 CNRS/Ecole Polytechnique Institut Polytechnique de Paris/ Sorbonne Université/Université Paris Saclay/Observatoire de Paris, Paris, France, ${ }^{14}$ National Space Science Center, State Key Laboratory of Space Weather, Chinese Academy of Sciences, Beijing, China, ${ }^{15}$ Department of Applied Physics, Universitat de Valencia, Valencia, Spain, ${ }^{16}$ Department of Applied Physics, University of Granada, Granada, Spain, ${ }^{17}$ Department of Electromagnetism and Matter Physics, University of Granada, Granada, Spain
\end{abstract}

\section{Introduction}

Electromagnetic ion cyclotron (EMIC) waves are generated in various regions of the Earth's magnetosphere when hot (keV to tens of keV) ions have $T_{\perp}>T_{\|}$(e.g., Gary, 1992; Gary \& Winske, 1990; Kennel \& 
Petschek, 1966). The wave growth rate maximizes in regions of $\mathbf{B}$ minima (e.g., Allen et al., 2015). EMIC waves are thought to grow at parallel wave normal angles $\left(\theta_{B k}\right)$ and exhibit left-handed polarization (LHP), but it is common to observe them propagating with large $\theta_{B k}$, and this is associated with a departure from LHP (e.g., Allen et al., 2015; Min et al., 2012).

One possible way of departing from LHP is propagation near the crossover frequency when heavy ions are present (Denton et al., 1996). Oblique propagation $\left(\theta_{B k}>30^{\circ}\right)$ is generally associated with small ellipticity and right-handed polarization (RHP) (Anderson et al., 1996). Hu and Denton (2009); Omidi et al. (2011) showed that propagation along the $\mathbf{B}$ field gradients of the Earth's dipole leads to oblique propagation of EMIC waves due to the changing refraction index and that the waves are reflected when they reach the local bi-ion frequency. However, for oblique propagation, it is expected that the wave is strongly damped (Thorne \& Horne, 1993). Anderson et al. (1992) observed that most EMIC waves in the dawn-sector exhibited small ellipticity that could not be explained only by propagation near the crossover frequency along a magnetic field gradient. Hu et al. (2010) showed, using 2.5D hybrid simulations, that the waves could be generated at oblique angles, in particular when there is a small amount of heavy ions and a large amount of cold protons, in addition to hot anisotropic protons which provide the energy source.

The magnetospheric multiscale (MMS) mission (Burch et al., 2015) provides unprecedented high-resolution measurements in the near-Earth plasma environment which have enabled studying the kinetic interaction of cold and hot protons in detail, and have recently shown the cold proton ability to remain magnetized inside spatial structures larger than their gyroradius (Alm et al., 2019; André et al., 2016; Shi et al., 2020; Toledo-Redondo et al, 2016, 2018).

In this work, we observe an EMIC wave propagating with a very oblique wave vector, and show that hot and cold protons interact with the wave electromagnetic fields in a kinetic and fluid sense, respectively. The cold protons have a gyroradius well below the observed perpendicular wavelength, allowing them to remain frozen-in and follow the fluctuations imposed by the slowly varying fields of the waves, exchanging energy adiabatically and favoring wave propagation at oblique angles.

\section{EMIC Wave Environment}

On October 24, 2015, at 15:26 UT, the MMS fleet (Burch et al., 2015) was in the dayside magnetosphere at $(7.3,8.0,-0.8)$ Earth radii $\left(\mathrm{R}_{E}\right)$ in geocentric solar ecliptic $(\mathrm{GSE})$ coordinates $\left(\mathrm{MLAT}=-23^{\circ}, \mathrm{L}\right.$-shell $\left.=12.8\right)$ and crossed the magnetopause multiple times. When the fleet re-entered the magnetosphere, it observed a wave for $\sim 20$ s. Figure 1a shows the magnetic field in GSE coordinates (Russell et al., 2014). From 15:27:25 UT onwards, marked by yellow shading, B fluctuations caused by the wave are observed. Figure 1b shows the electric field measurements in GSE coordinates (Ergun et al., 2014; Lindqvist et al., 2014). Electric fields of $\sim 10 \mathrm{mV} / \mathrm{m}$ consistent with separatrix crossings are observed on the magnetospheric edges of the magnetopause. Electric field fluctuations associated with the wave are observed from 15:27:25 UT onwards. Figure 1c shows the total ion (black), electron (blue), $\mathrm{He}^{+}$(red), $\mathrm{He}^{2+}$ (green), and $\mathrm{O}^{+}$(gray) number densities recorded by the fast plasma investigation (FPI) (Pollock et al., 2016) and the hot plasma composition analyzer (HPCA) (Young et al., 2014). The total density in the magnetosphere is roughly $1 \mathrm{~cm}^{-3}$, mainly contributed by cold and hot protons. The measured electron density goes below $1 \mathrm{~cm}^{-3}$ and deviates from the ion density toward the end of the interval. The reason is likely the presence of cold electrons below the $10 \mathrm{eV}$ threshold of FPI. During the entire interval of Figure 1, the spacecraft was charged positively below $10 \mathrm{~V}$. Figure $1 \mathrm{~d}$ shows the ion velocity (GSE) recorded by FPI. We observe an ion flow in the $-\boldsymbol{z}_{\mathrm{GSE}}$ direction that peaks at $-250 \mathrm{~km} / \mathrm{s}$, corresponding to $1.1 v_{A}$, where $v_{A}$ is the observed hybrid Alfvén velocity at the magnetopause (Cassak \& Shay, 2007). The ion flow and the electric field separatrix signatures indicate that reconnection may be occurring at the magnetopause, with the $\mathrm{X}$ line located northward of the spacecraft, consistent with the maximum shear model predictions at that time (Trattner et al., 2007). At the end of the time interval, the magnetopause is moving sunward at a peak velocity of $\sim 150 \mathrm{~km} / \mathrm{s}$. Figure 1 e shows an ion energy spectrogram, where three populations can be distinguished. In the magnetosphere, there is a hot population with energies above $2 \mathrm{keV}$, the plasma sheet ions, plus a cold population with measured energies of 50-300 eV (bulk velocity plus thermal energies), of ionospheric origin. The black line is the equivalent $\mathbf{E}$ $\times \mathbf{B}$ energy for protons and indicates the energy associated with the perpendicular bulk velocity of the cold 


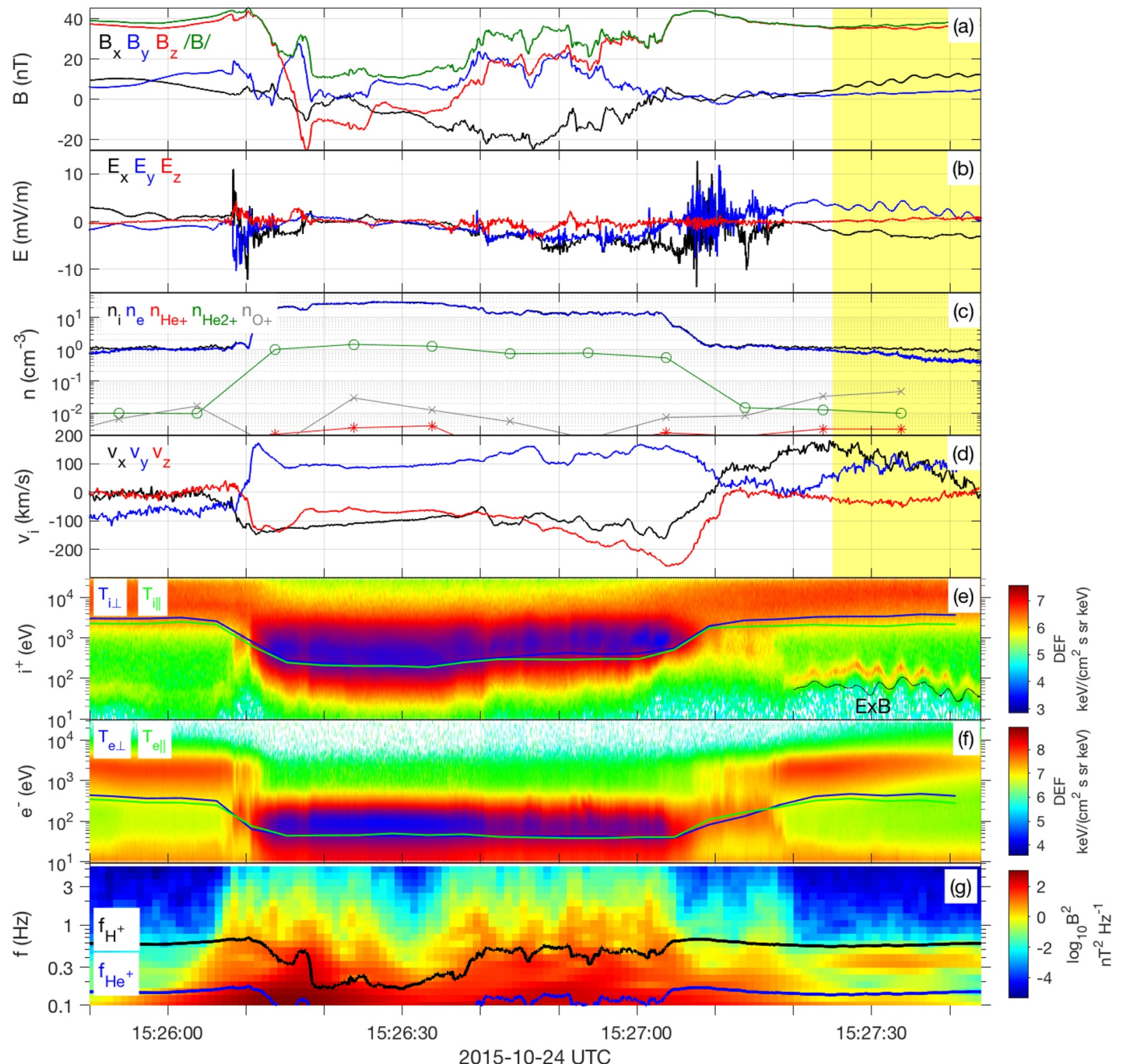

Figure 1. Overview of the MMS1 magnetopause crossing. (a) Magnetic field in GSE coordinates. (b) Electric field in GSE coordinates. (c) (black) Number densities of all ions from FPI, (blue) electrons from FPI, (red, green, and gray), and heavy ions $\left(\mathrm{He}^{+}, \mathrm{He}^{2+}\right.$, and $\mathrm{O}^{+}$) from HPCA. (d) FPI ion velocity in GSE coordinates. (e) (color) FPI ion differential energy flux (DEF), (black) equivalent $\mathbf{E} \times \mathbf{B}$ energy for protons, (blue) perpendicular ion temperature ( $\left.T_{i \perp}\right)$, (green) parallel ion temperature $\left(T_{i \|}\right)$. (f) FPI electron DEF, (blue) perpendicular electron temperature $\left(T_{e \perp}\right)$, (green) parallel electron temperature $\left(T_{e \|}\right)$. (g) (color) Magnetic field spectrogram, (black) $\mathrm{H}^{+}$cyclotron frequency, (blue) $\mathrm{He}^{+}$cyclotron frequency. FPI, fast plasma investigation; GSE, geocentric solar ecliptic; HPCA, hot plasma composition analyzer; MMS, magnetospheric multiscale.

ions in the spacecraft frame. The third one is the ion population with energies from a few tens of eV up to a few keV from the magnetosheath. The total parallel $\left(T_{i \|}\right)$ and perpendicular $\left(T_{i \perp}\right)$ temperatures are shown using green and blue lines, respectively. The cold ion heating observed between 15:27:10 and 15:27:20 UT has been previously studied by Toledo-Redondo et al. (2017). From 15:27:20 UT onwards, the cold ion energy fluctuates up and down as a consequence of the interaction with the wave. Figure $1 \mathrm{f}$ shows the electron energy spectrogram recorded by FPI dual electron spectrometers (DES). As for the ions, three populations can be distinguished based on their energies: plasma sheet electrons, cold electrons of ionospheric origin, and magnetosheath electrons. Figure $1 \mathrm{~g}$ shows the magnetic field dynamic spectrum in the low frequency (0.1-6 Hz) band. The magnetic field fluctuations observed after 15:27:20 UT have a peak in power at $\sim 0.35 \mathrm{~Hz}$ in the spacecraft frame, below the $\mathrm{H}^{+}$and above the $\mathrm{He}^{+}$cyclotron frequency bands. 


\section{Observed Wave Properties}

We now focus on the low-frequency wave observation $\left(f_{s c} \sim 0.35 \mathrm{~Hz}\right)$ in the yellow-shaded interval of Figures 1, 15:27:25-15:27:44 UT. The MMS fleet is in tetrahedron formation with a spacecraft separation of $\sim 15 \mathrm{~km}$, much smaller than the characteristic wavelength $(\lambda)$ of the wave under study (see below), and all quantities in Figure 2 represent 4-spacecraft averages. Figure 2a shows the ion energy spectrogram recorded by FPI in the low-energy range. The equivalent $\mathbf{E} \times \mathbf{B}$ energy for protons is plotted in black. The energy of the cold ion population fluctuates periodically between tens of $\mathrm{eV}$ and a few hundred eV. For most of the interval, the average energy of the cold ions is above $50 \mathrm{eV}$, except for the last 3-4 s. Therefore, the FPI-ion and $\mathbf{E}$ field measurements are in general only weakly affected by the sheath electrostatic potential of the spacecraft and the formation of cold ion wakes, except for the last 3-4 s, where the effect may be substantial (Toledo-Redondo et al., 2019).

We computed 4-spacecraft-averaged partial moments $(n, \mathbf{v}, \mathbf{P})$ for the cold ion (subscript ic) and hot ion (subscript $i h)$ populations. Parallel and perpendicular temperatures $\left(T_{\|}, T_{\perp}\right)$ correspond to the diagonal elements of the tensor $\mathbf{T}=\mathbf{P} /\left(n K_{b}\right)$, rotated into field-aligned coordinates (see below), where $K_{b}$ is the Boltzmann constant. We consider the FPI ion distribution functions in the interval (10-400) eV for cold ions and (2-40) $\mathrm{keV}$ for hot ions. More details on these calculations can be found in for example, Lee et al. (2019, 2021), Li et al. (2017), and Toledo-Redondo et al. (2016).

Figure 2b shows the electron density fluctuations $\left(\Delta n_{e}\right)$ from FPI, and the partial cold and hot ion density fluctuations $\left(\Delta n_{i c}, \Delta n_{i h}\right)$. Density fluctuations $(\Delta n)$ are computed using a fifth order elliptical band-pass filter, with cutoff frequencies at $0.1 f_{H^{+}}$and $5 f_{H^{+}}$, where $f_{H^{+}}=0.57 \mathrm{~Hz}$, corresponding to the proton cyclotron frequency in the interval 15:27:25-15:27:44 UT. Fluctuations $(\Delta)$ of any quantity throughout the study are computed using the same filtering. The total ion and electron density is $\sim 1 \mathrm{~cm}^{-3}$ (Figure 1c). The number density of the heavy ion species contributes less than $10 \%$, and most of the ions correspond to protons, of which approximately one-half corresponds to hot protons and one half to cold protons (not shown). There is a fluctuation of the electron and cold proton density of $\sim 0.1 \mathrm{~cm}^{-3}$ (i.e., $20 \%$ of the cold proton density) that is not observed for the hot protons.

The average direction of $\mathbf{B}$ in the time interval of Figure 2 defines $\hat{\mathbf{e}}_{\|}=(0.26,0.09,0.96)$ in GSE. We apply maximum variance analysis (MVA) to $\Delta \mathbf{B}$ and obtain $\hat{\mathbf{u}}_{\max , \Delta B}=(0.97,0.01,-0.23)$ for the maximum variance direction. We define $\hat{\mathbf{e}}_{\perp 2}=\hat{\mathbf{e}}_{\|} \times \hat{\mathbf{u}}_{\max }=(-0.03,0.99,-0.09)$, and $\hat{\mathbf{e}}_{\perp 1}=(0.96,0.01,-0.26)$ in GSE closes the system.

The system $\left(\hat{\mathbf{e}}_{\|}, \hat{\mathbf{e}}_{\perp 1}, \hat{\mathbf{e}}_{\perp 2}\right.$ ) defines the field-aligned coordinates (FAC) used in this study. We note that $\hat{\mathbf{e}}_{\perp 2}$ is within less than $6^{\circ}$ of $\hat{\mathbf{u}}_{\max , \Delta E}$, that is, the maximum variance direction of $\Delta \mathbf{E} . \Delta \mathbf{B}$ and $\Delta \mathbf{E}$ are plotted in Figures $2 \mathrm{c}$ and $2 \mathrm{~d}$, respectively. The black vertical lines in Figure 2 indicate $\Delta B_{\perp 1}$ maxima. $\Delta \mathbf{B}$ exhibits small ellipticity and RHP, with L2/L1 $\sim 0.26$, where L2 and L1 are the eigenvalues of the intermediate and maximum directions obtained by MVAB, respectively. Cold $\left(\Delta \mathbf{v}_{i c}\right)$ and hot $\left(\Delta \mathbf{v}_{i h}\right)$ ion velocity fluctuations are plotted in Figures 2e and 2f. The perpendicular $\Delta \mathbf{v}_{i c}$ components clearly fluctuate with the electromagnetic fields, in contrast to $\Delta \mathbf{v}_{i h}$.

We compute the fluctuations of the Ohm's law terms, for a three fluid plasma including electrons, cold protons and hot protons (Toledo-Redondo et al., 2015):

$$
\Delta \mathbf{E}=-\Delta\left(\frac{n_{i c}}{n} \mathbf{v}_{\mathbf{i c}} \times \mathbf{B}\right)-\Delta\left(\frac{n_{i h}}{n} \mathbf{v}_{\mathbf{i h}} \times \mathbf{B}\right)+\Delta\left(\frac{1}{e n} \mathbf{J} \times \mathbf{B}\right)-\Delta\left(\frac{1}{e n} \nabla \cdot \mathbf{P}_{\mathbf{e}}\right)
$$

where $\mathbf{J}$ was obtained using the curlometer technique (Dunlop et al., 1988). The heavy ion convection terms can be neglected due to their small number densities. Inside the magnetosphere, the electron density is small $\left(\sim 1 \mathrm{~cm}^{-3}\right)$ and the electron temperature is large (hundreds of eV), and we cannot reliably obtain the $\nabla \cdot \mathbf{P}_{e} /$ en term, although we expect it to be small. Although MMS observed two electron populations in the magnetosphere, we treat them as a single population for simplification, since we do not expect a differential behavior of the two populations at the time and spatial scales of the wave. This is confirmed using a wave 

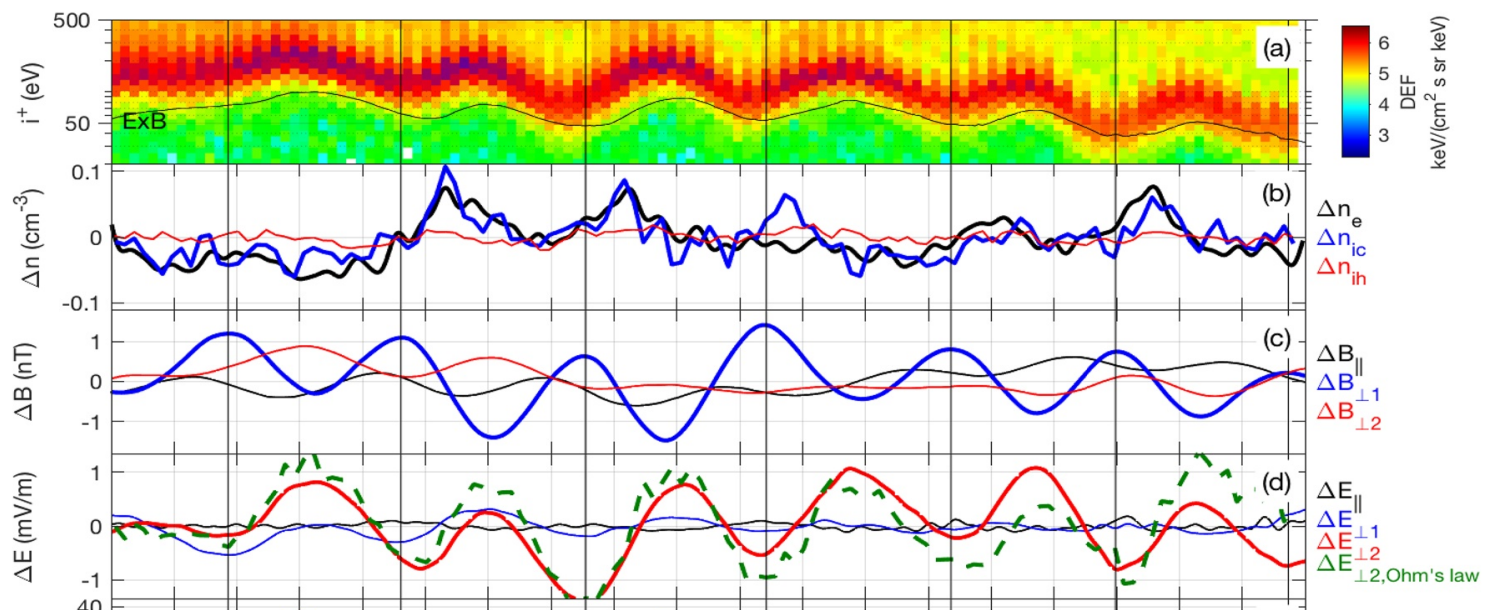

$\underset{-20}{20}$

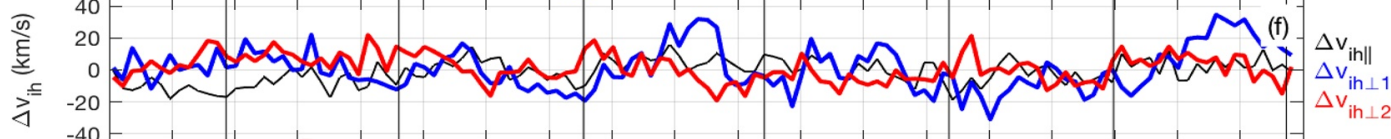
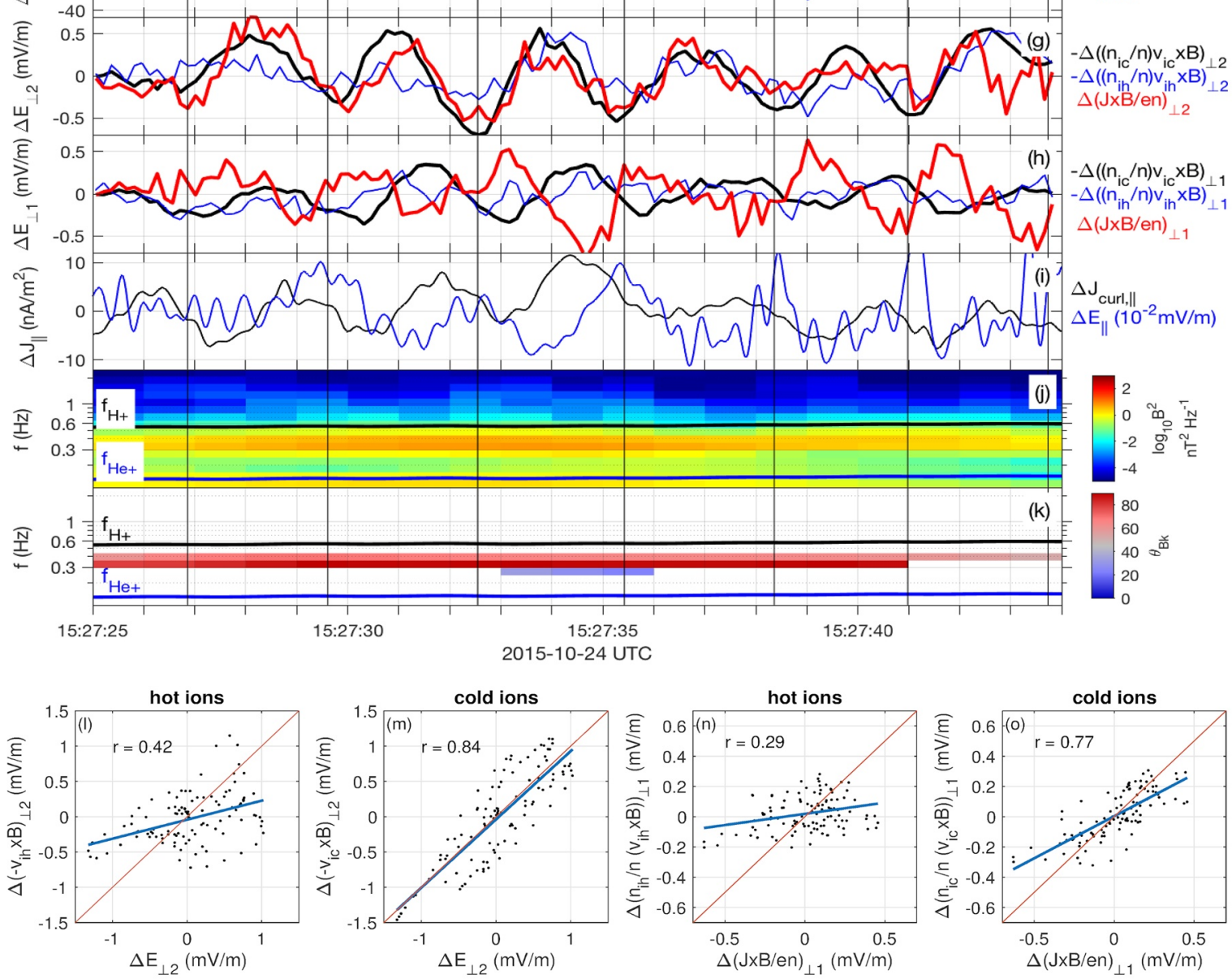
dispersion solver, which yielded the same results for the Alfvén branch when accounting for a single or double electron population (cf. Section 3).

The $\hat{\mathbf{e}}_{\perp 2}$ components of the fluctuations of the Ohm's law right-hand side terms are plotted in Figure $2 \mathrm{~g}$. The main contributions are provided by the cold ion convection term and the Hall term, and to a lesser degree by the hot ion convection term. The sum of the right-hand side terms of Equation 1 is also plotted in Figure $2 \mathrm{~d}$ (green dashed line). The agreement between the measured electric field fluctuations and the sum of the right-hand side terms of Equation 1 is very good, except for the last $3 \mathrm{~s}$ of the time interval of Figure 2 when the cold ion energy is lower and both $\mathbf{E}$ and FPI-ion measurements become less reliable owing to the electrostatic potential structure of the spacecraft and ion wake effects (Toledo-Redondo et al., 2019). We performed a linear regression analysis between $\Delta \mathbf{E}$ and $-\Delta \mathbf{v}_{\mathbf{i c}} \times \mathbf{B}$ in the $\hat{\mathbf{e}}_{\perp 2}$ direction, and found a correlation coefficient $r=0.84$ (Figure 2i), for the time interval of Figure 2 excluding the last $3 \mathrm{~s}$, while the correlation between $\Delta E_{\perp 2}$ and $-\Delta\left(\mathbf{v}_{\mathbf{i h}} \times \mathbf{B}\right)_{\perp 2}$ was $r=0.42$ (Figure 21). This suggests that cold ions are magnetized and follow $\mathbf{E} \times \mathbf{B}$ motion, while hot ions are less magnetized. Figure $2 \mathrm{~h}$ shows the Ohm's law terms in the $\hat{\mathbf{e}}_{\perp 1}$ direction. The net $\Delta E_{\perp 1}$ field is negligible $\left(\Delta E_{\perp 1} \sim 0.1 \Delta E_{\perp 2}\right)$ (blue and red curves in Figure 2d), consistent with the small wave ellipticity. This results from the nonnegligible contributions of the cold ion convection term and the Hall term in the $\perp 1$ direction (black and red curves in Figure 2h), which roughly cancel each other. The correlation coefficient between the fluctuations of the cold ion convection term, $\Delta\left(n_{i c} / n\left(\mathbf{v}_{\mathbf{i c}} \times \mathbf{B}\right)\right)_{\perp 1}$ (black curve in Figure $2 \mathrm{~h}$ ), and the Hall term, $\Delta(\mathbf{J} \times \mathbf{B} / e n)$ (red curve in Figure $2 \mathrm{~h}$ ), in the $\hat{\mathbf{e}}_{\perp 1}$ direction is $r=0.77$ (Figure 20), while the correlation between $\Delta\left(n_{i h} / n\left(\mathbf{v}_{\mathbf{i h}} \times \mathbf{B}\right)\right)_{\perp 1}$ and $\Delta(\mathbf{J} \times \mathbf{B} / \text { en })_{\perp 1}$ is 0.29 (Figure $2 \mathrm{n})$. We compute the associated speed of the field fluctuations $\operatorname{RMS}(\Delta E / \Delta B=750 \mathrm{~km} / \mathrm{s})$, where RMS stands for root mean squared. The associated Alfvén velocity of the interval is $v_{A}=770 \mathrm{~km} / \mathrm{s}(B=36 \mathrm{nT}$, $n=1 \mathrm{~cm}^{-3}$ ), indicating that the wave likely corresponds to the Alfvénic branch.

The currents are calculated using two methods: the curlometer, and 4-spacecraft-averaged plasma moments. The two methods yield similar results (not shown). Figure 2i shows $\Delta J_{\|}$using curlometer (black), and $\Delta E_{\|}$ (blue). The parallel current is roughly at $90^{\circ}$ phase shift with respect to $\Delta E_{\|}$, which results in a fluctuating contribution to J · E (Gershman et al., 2017; Hollweg, 1999). Figure 2j shows a magnetic field spectrogram. The wave power is located between the $\mathrm{He}^{+}$(blue line) and the $\mathrm{H}^{+}$(black line) cyclotron bands, at $\sim 0.35 \mathrm{~Hz}$ in the spacecraft frame, see also Figure 1g. Magnetic field polarization analysis shows that the angle between the wave vector $\mathbf{k}$ and the background magnetic field, $\theta_{B k}$ is $\sim 70^{\circ}$ (Figure 2k). Bellan (2016) presented a method to compute the $\mathbf{k}$ vector of low-frequency waves if the current density vector $\mathbf{J}$ is known. It is based on the Ampere's law in the frequency domain, assuming a monochromatic wave: $\mu_{0} \mathbf{J}(\omega)=i \mathbf{k}(\omega)$ $\times \mathbf{B}(\omega)$. Following that procedure and calculating the fluctuations of the current density vector $\Delta \mathbf{J}$ using the curlometer technique, we obtain $\mathbf{k}_{\text {Bellan }}=(1.6,0.6,5.7) \cdot 10^{-3} \mathrm{rad} / \mathrm{km}$ in $\operatorname{FAC}\left(\hat{\mathbf{e}}_{\|}, \hat{\mathbf{e}}_{\perp 1}, \hat{\mathbf{e}}_{\perp 2}\right)$. We confirm the $\mathbf{k}_{\text {Bellan }}$ computation using a similar and recently proposed method, the wave curl analysis (Vines et al., 2021). We also compute the $\mathbf{k}$ vector from four-spacecraft cross-correlations and time differencing analysis of the magnetic field (Balikhin et al., 2003; Pinçon \& Glassmeier, 2008). We obtain a very similar result, $\mathbf{k}_{4 s c}=(2.0,0.83,5.0) \cdot 10^{-3} \mathrm{rad} / \mathrm{km}$ in FAC, corresponding to a difference of roughly $7^{\circ}$ from $\mathbf{k}_{\mathrm{Bellan}}$. We assumed the wave to be monochromatic with a frequency of $0.35 \mathrm{~Hz}$ in the spacecraft frame, corresponding to the frequency where the magnetic field spectrum peaks. More details of these calculations can be found in Figure S1. We conclude that the angle between $\mathbf{B}$ and $\mathbf{k}$ is $\theta_{B k} \sim 74^{\circ}$, as indicated by three independent methods. The median bulk ion velocity during the interval of the wave observation, 15:27:25-15:27:44 UT, is $\mathbf{v}_{0}=(98,95,-27) \mathrm{km} / \mathrm{s}$ in GSE. After correction for the doppler shift effect $\left(f_{\text {wave }}=f_{s c}-\mathbf{k} \cdot \mathbf{v}_{0} / 2 \pi\right)$, the frequency of the wave in the plasma frame is found to be $f_{\text {wave }}=0.26 \mathrm{~Hz}$, that is, roughly $0.5 f_{H^{+}}$.

Figure 2. EMIC wave observation in the interval 15:27:25 UT-15:27:44 UT. All panels correspond to 4-spacecraft averages. Vertical black lines indicate the peaks in $\Delta B_{\perp 1}$. (a) (color) FPI Ion energy spectrogram in DEF, (black) equivalent $\mathbf{E} \times \mathbf{B}$ energy for protons, (b) Density fluctuations for electrons ( $\Delta n_{e}$, black), cold magnetospheric ions $\left(\Delta n_{i c}\right.$, blue), and hot magnetospheric ions $\left(\Delta n_{i h}\right.$, red). (c) Magnetic field fluctuations $(\Delta \mathbf{B})$ in FAC. (d) Electric field fluctuations ( $\left.\Delta \mathbf{E}\right)$ in FAC and sum of the right-hand side terms of Equation 1 for the $\hat{\mathbf{e}}_{\perp 2}$ direction. (e) Cold ion velocity fluctuations ( $\Delta \mathbf{v}_{i c}$ ) in FAC. (f) Hot ion velocity fluctuations $\left(\Delta \mathbf{v}_{i h}\right)$ in FAC. (g) Ohm's law terms for the $\hat{\mathbf{e}}_{\perp 2}$ direction. (h) Ohm's law terms for the $\hat{\mathbf{e}}_{\perp 1}$ direction. (i) Parallel component of the current density fluctuations estimated using the curlometer technique $\Delta \mathbf{J}$ (black), and parallel electric field fluctuation (blue). (j) Magnetic field power spectral density measured by MMS1 near the $\mathrm{H}^{+}$and $\mathrm{He}^{+}$cyclotron frequencies (black and blue lines, respectively). (k) Angle between magnetic field and wave vector, $\theta_{B k}$, for power spectral densities $>1 \mathrm{nT}^{2} \mathrm{~Hz}^{-1}$. (1) Linear regression analysis of $\Delta \mathbf{E}$ and $-\Delta\left(\mathbf{v}_{i n} \times \mathbf{B}\right)$ in the $\hat{\mathbf{e}}_{\perp 2}$ direction. (m) Linear regression analysis of $\Delta \mathbf{E}$ and $-\boldsymbol{\Delta}\left(\mathbf{v}_{i c} \times \mathbf{B}\right)$ in the $\hat{\mathbf{e}}_{\perp 2}$ direction. (n) Linear regression analysis of $\Delta(\mathbf{J} \times \mathbf{B} /$ en $)$ and $\Delta\left(n_{i h} / n \mathbf{v}_{i h} \times \mathbf{B}\right)$ in the $\hat{\mathbf{e}}_{\perp 1}$ direction. (o) Linear regression analysis of $\Delta(\mathbf{J} \times \mathbf{B} /$ en $)$ and $\Delta\left(n_{i c} / n \mathbf{v}_{i c}\right.$ $\times \mathbf{B})$ in the $\hat{\mathbf{e}}_{\perp 1}$ direction. DEF, differential energy flux; EMIC, electromagnetic ion cyclotron; FAC, field-aligned coordinates; FPI, fast plasma investigation. 


\section{Modeled Wave Properties}

Next, we model the wave using waves in homogeneous anisotropic magnetized plasma (WHAMP) (Roennmark, 1982), accounting for the populations measured by MMS: $\mathrm{O}^{+}, \mathrm{He}^{2+}, \mathrm{He}^{+}$, cold $\mathrm{H}^{+}$, hot $\mathrm{H}^{+}$, and electrons. Their density, temperature, and anisotropy are taken from the average value in the time interval of Figure 2. There is no strong background current during the event, so the relative drift velocities between populations are set to zero for all species and there are no ion-ion instability effects. The average plasma parameters of each population can be found in Table S1. Accounting for a cold electron population has no significant effects over the Alfvén branch. The results of the dispersion solver for the Alfvén branch near $f_{H^{+}}$, including five ion populations plus electrons, are shown in Figures 3a-3d. Panel 3a shows the normalized frequency $\left(\Omega / \Omega_{i}\right)$, where $\Omega_{i}=2 \pi f_{H^{+}}$, as a function of normalized $k_{\|}\left(k_{\|} \rho_{i h}\right)$, where $\rho_{i h}$ is the hot ion gyroradius, for $k_{\perp} \rho_{i h}=0$ and 1.98 (red and black lines), corresponding to $\theta_{B k}=0^{\circ}$ and $74^{\circ}$ at the measured $k_{\|}$, respectively. The green asterisk corresponds to the normalized frequency measured by MMS and corrected for Doppler shift, which is within 7\% of the prediction by WHAMP for the same $\mathbf{k}$ vector and within $12 \%$ of the frequency for the predicted maximum growth rate. Figure $3 \mathrm{~b}$ is similar to Figure $3 \mathrm{a}$, but the vertical axis represents the normalized growth rate $\left(\gamma / \Omega_{i}\right)$. The growth rate is maximum for $\theta_{B k}=0^{\circ}$, and becomes slightly negative at the measured wave normal angle $\theta_{B k}=74^{\circ}$. Figure $3 \mathrm{c}$ shows the growth rate along the dispersion surface of the Alfvén branch. For the observed frequency (green asterisk) and $\theta_{B k}$, the wave is slightly damped, but we note that for $\theta_{B k} \leq 50^{\circ}$ the growth rate becomes positive. Figure $3 \mathrm{~d}$ is similar to Figure $3 \mathrm{c}$ but the colormap indicates the wave ellipticity $\epsilon=\operatorname{Re}\left(i B_{\perp 2} / B_{\perp 1}\right)$. Values close to 1 indicate circular RHP. The dispersion solver predicts an ellipticity $\epsilon=0.23$, that is, within $\sim 10 \%$ of the measured ellipticity. Figures 3a-3d suggest that the measurement did not occur in the wave source region, but locally the wave can propagate without strong damping.

We present three runs with varying amounts of cold $\left(T_{i c}=40 \mathrm{eV}\right)$ proton density, $n_{i c}=[0.1,0.5,1] \mathrm{cm}^{-3}$, in Figures $3 \mathrm{e}-3 \mathrm{~g}$, where the hot proton population has been left unchanged, and the electron population provides quasi-neutrality. For simplicity, we did not include heavy-ion populations in these runs. The hot proton parameters for the three runs are $n_{i h}=0.5 \mathrm{~cm}^{-3}, T_{\|}=4.4 \mathrm{keV}$, and $T_{\perp} / T_{\|}=1.8$. The complete description of the plasma parameters is provided in Table S2. The positive growth rate is larger and occurs over larger frequency and $\mathbf{k}$ vector ranges when more cold protons are present, despite that the source of energy, i.e., hot proton temperature anisotropy, remains constant (Gary et al., 1994). The largest growth rate is observed for small $k_{\perp} \rho_{i h}$ and $k_{\|} \rho_{i h} \sim 0.5$ in the three runs, but large positive growth rates are present for large $k_{\perp} \rho_{i h}$ when cold $\mathrm{H}^{+}$density becomes significant (Figures $3 \mathrm{f}$ and $3 \mathrm{~g}$ ). The run with $n_{i c}=1 \mathrm{~cm}^{-3}$ has positive growth rates for $\theta_{B k}$ up to $74^{\circ}$ and shows small ellipticity and RHP at these large wave normal angles.

\section{Discussion and Conclusions}

The measured k vector, Doppler-shifted frequency, and ellipticity are in good agreement with the dispersion relation predicted by the numerical solver. The solver also indicates that the wave is not strongly damped at the observed $\mathbf{k}$ vector, although the maximum growth rate is expected for $\mathbf{k}$ close to parallel, suggesting that the observation may be out of the source region. We cannot conclude from the observation if the wave was generated with parallel $\mathbf{k}$ vector and then became oblique during propagation, or if the wave was directly generated with oblique $\mathbf{k}$ vector. For instance, propagation across magnetic field and density gradients can result in large $\theta_{B k}$ (e.g., Hu \& Denton, 2009; Omidi et al., 2011; Thorne \& Horne, 1993). These studies did not include a cold proton component and the wave was expected to be strongly damped for large $\theta_{B k}$, but our comparison of three runs varying the cold proton number density indicates that cold protons reduce wave damping and even result in positive growth rates for propagation at large wave normal angles, consistent with the hybrid simulations by Hu et al. (2010). This way of producing wave growth at oblique angles can explain the observations by Anderson et al. (1992).

A careful examination of the $\mathbf{E}$ field fluctuations and the contributions by the Ohm's law terms reveal that cold protons are fully magnetized while hot protons are, to a certain extent, demagnetized. The fluctuations of the cold proton term, $-\Delta n_{i c} / n\left(\mathbf{v}_{i c} \times \mathbf{B}\right)$, have LHP, while the fluctuations of the Hall term, $\Delta(\mathbf{J} \times \mathbf{B} /$ en), have RHP (see Figures $2 \mathrm{~g}$ and $2 \mathrm{~h}$ ). The contributions of these two terms result in the observed small 
Alfvén branch for the plasma parameters observed by MMS (15:27:25 UT - 17:27:44 UT)
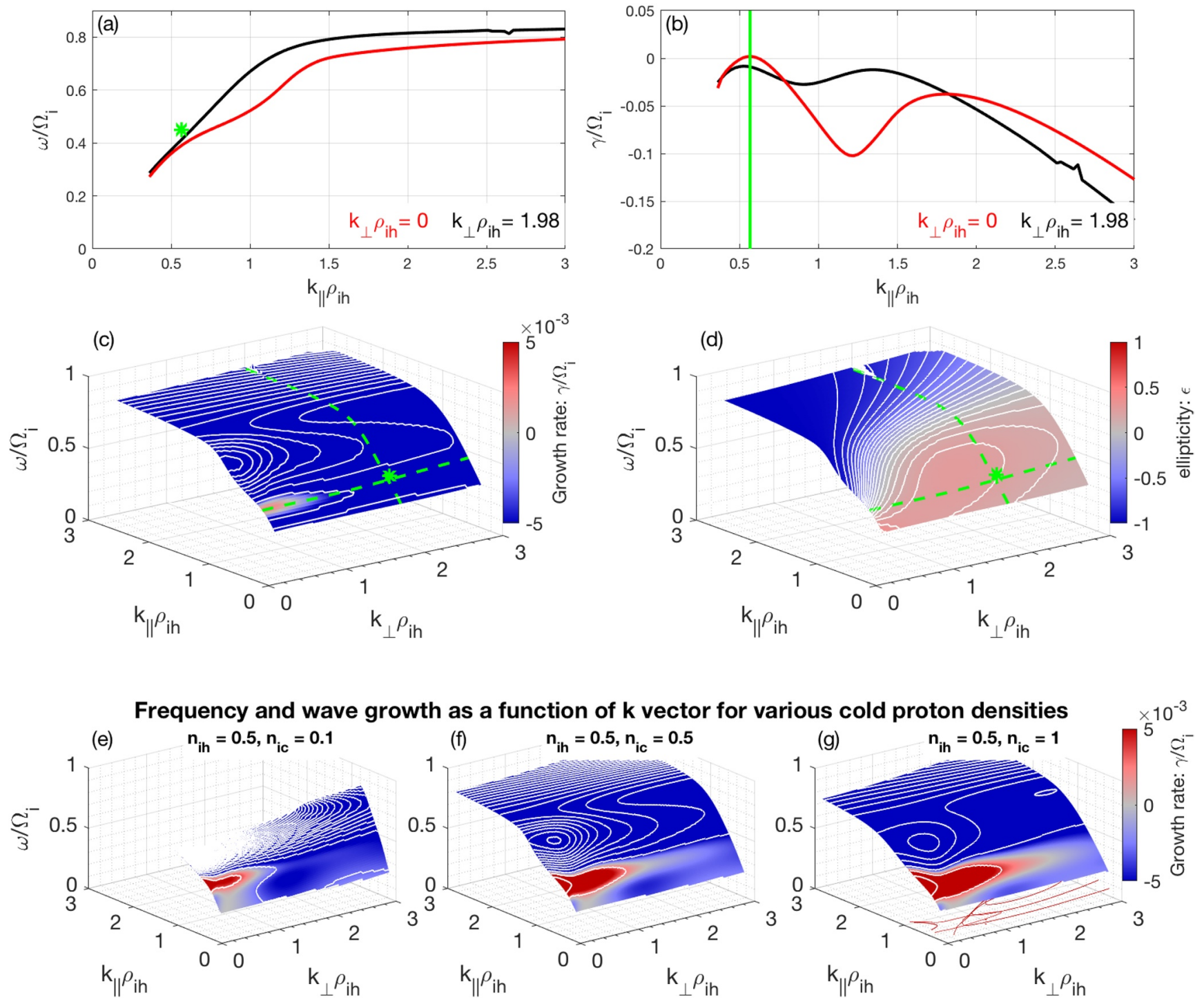

Figure 3. (a-d) Dispersion relation of the Alfvén branch corresponding to the plasma parameters measured by MMS in the interval 15:27:25-15:27:44 UT. The plasma parameters are specified in Table S1. (a) Normalized frequency $\left(\Omega / \Omega_{i}\right)$ as a function of the normalized parallel component of the wavevector $\left(k_{\|} \rho_{i h}\right)$, for $k_{\perp} \rho_{i h}=0$ (red), and the observed $k_{\perp} \rho_{i h}=1.98$ (black). The green asterisk indicates the wave frequency in the plasma rest frame measured by MMS. (b) Same as (a) for the growth rate instead of frequency. The green line indicates the measured $k_{\|, \text {Bellan. }}$. (c) Alfvén branch dispersion surface. The colorbar indicates normalized growth rate $\left(\gamma / \Omega_{i}\right)$. The green asterisk indicates the wave frequency in the plasma rest frame and $\mathrm{k}_{\|}$measured by MMS. (d) Same as (c) but the colorbar indicates the ellipticity, $\epsilon=\operatorname{Re}\left(\mathrm{i} B_{\perp 2} / B_{\perp 1}\right)$. (e-g) Dispersion relations and normalized growth rate of the Alfvén branch for different amounts of cold protons, keeping the hot proton population unchanged. Heavy ions are excluded, for simplicity, in these runs. The plasma parameters are specified in Table S2. MMS, magnetospheric multiscale.

ellipticity of $\Delta \mathbf{E}$. The physical picture is that the cold protons follow the perpendicular fluctuations of the fields, exchanging energy back and forth with them adiabatically and sustaining wave propagation. On the other hand, the hot proton gyration is comparable to the perpendicular wavelength and the interaction is nonadiabatic.

Three characteristic length scales are considered for protons: the proton inertial length $\left(d_{i}\right)$, the cold proton gyroradius $\left(\rho_{i c}\right)$, and the hot proton gyroradius $\left(\rho_{i h}\right)$. We compare them to the wavenumber and find $k_{\perp} d_{i}=1.4, k_{\perp} \rho_{i c}=0.12$, and $k_{\perp} \rho_{i h}=1.9$. Only the cold proton gyroradius is significantly smaller than the characteristic scale of the wave, and this would explain why the hot protons are, to a large extent, demagnetized (note, however, that the demagnetization of the hot protons is not fully achieved; see Figures $2 \mathrm{~g}$ 
and 21). The ratio $k_{\perp} \rho_{i c} \ll 1$ is consistent with the observed cold proton magnetization, indicating that cold proton gyration occurs at a scale much smaller than the perpendicular wavelength. It is interesting to see that cold protons remain fully frozen-in, despite $k_{\perp} d_{i}=1.4$. We expect that cold protons would also be demagnetized for larger $k_{\perp} d_{i}$. Since the cold protons remain frozen-in, it is not expected that they will be significantly heated, consistent with the observations by Anderson and Fuselier (1994). In summary, the wave-proton interaction is in a hybrid regime, with the cold proton population interacting as a fluid and the hot proton population interacting kinetically.

The wave was observed very close to the reconnecting magnetopause, and therefore it is likely that the source of energy was compressions of the magnetosphere driven by solar wind pressure pulses, resulting in the observed hot ion temperature anisotropy (e.g., Anderson \& Hamilton, 1993; Engebretson et al., 2015). These waves can, in turn, accelerate and heat some of the magnetospheric ion populations, particularly heavy ions (e.g., Tanaka, 1985; Zhang et al., 2011), potentially acting as a preconditioning process of the plasma inflowing toward the reconnecting magnetopause.

We showed detailed 4-spacecraft measurements inside an EMIC wave near the reconnecting magnetopause reconnection and provided observational evidence of the different dynamics of cold and hot protons. They interact in a fluid and kinetic fashion, respectively, and this has implications for the electric fields and currents that the wave sets, favoring wave propagation and growth at oblique angles, with small ellipticity and RHP. The cold proton population exchanges energy with the fields adiabatically and supports wave propagation, while the hot protons cannot follow the fluctuations due to the small perpendicular wavelength. This provides a possible explanation for the predominance of small ellipticity and RHP EMIC waves in the Earth's magnetosphere (e.g., Anderson et al., 1992; Min et al., 2012; Allen et al., 2015), which is often populated by cold ions of ionospheric origin (e.g., André \& Cully, 2012).

\section{Data Availability Statement}

MMS data are publicly available at https://lasp.colorado.edu/mms/sdc/public/.

\section{Acknowledgments}

STR acknowledges support from the ISSI international team Cold plasma of ionospheric origin in the Earth's magnetosphere and of the Ministry of Economy and Competitiveness (MINECO) of Spain (grant FIS201790102-R). Research at IRAP was supported by CNRS, CNES and the University of Toulouse. JHL and DLT acknowledge support from NASA Grant 80NSSC18K1378. RED was supported by NASA grants $80 \mathrm{NSSC} 19 \mathrm{~K} 070$ and 80NSSC19K0254. MA was supported by SNSA Grant 56/18. SKV and RCA acknowledge support from NASA Grant 80NSSC19K0270. Work performed by MMS team members is supported by NASA contract NNG04EB99C.

\section{References}

Allen, R. C., Zhang, J.-C., Kistler, L. M., Spence, H. E., Lin, R.-L., Klecker, B., et al. (2015). A statistical study of emic waves observed by cluster: 1. wave properties. Journal of Geophysical Research: Space Physics, 120(7), 5574-5592. https://doi.org/10.1002/2015ja021333

Alm, L., André, M., Graham, D. B., Khotyaintsev, Y. V., Vaivads, A., Chappell, C. R., et al. (2019). Mms observations of multiscale hall physics in the magnetotail. Geophysical Research Letters, 46(17-18), 10230-10239. https://doi.org/10.1029/2019gl084137

Anderson, B. J., Denton, R. E., Ho, G., Hamilton, D. C., Fuselier, S. A., \& Strangeway, R. J. (1996). Observational test of local proton cyclotron instability in the Earth's magnetosphere. Journal of Geophysical Research, 101(A10), 21527-21543. https://doi.org/10.1029/96ja01251

Anderson, B. J., Erlandson, R. E., \& Zanetti, L. J. (1992). A statistical study of pc 1-2 magnetic pulsations in the equatorial magnetosphere: 2. wave properties. Journal of Geophysical Research, 97(A3), 3089-3101. https://doi.org/10.1029/91ja02697

Anderson, B. J., \& Fuselier, S. A. (1994). Response of thermal ions to electromagnetic ion cyclotron waves. Journal of Geophysical Research, 99(A10). https://doi.org/10.1029/94ja01235

Anderson, B. J., \& Hamilton, D. C. (1993). Electromagnetic ion cyclotron waves stimulated by modest magnetospheric compressions. Journal of Geophysical Research, 98(A7), 11369-11382. https://doi.org/10.1029/93ja00605

André, M., \& Cully, C. M. (2012). Low-energy ions: A previously hidden solar system particle population. Geophysical Research Letters, 39(3). https://doi.org/10.1029/2011gl050242

André, M., Li, W., Toledo-Redondo, S., Khotyaintsev, Y. V., Vaivads, A., Graham, D. B., et al. (2016). Magnetic reconnection and modification of the hall physics due to cold ions at the magnetopause. Geophysical Research Letters, 43(13), 6705-6712. https://doi.org/10.1002/2016gl069665

Balikhin, M. A., Pokhotelov, O. A., Walker, S. N., Amata, E., Andre, M., Dunlop, M., \& Alleyne, H. S. C. K. (2003). Minimum variance free wave identification: Application to cluster electric field data in the magnetosheath. Geophysical Research Letters, 30(10). https://doi. org/10.1029/2003gl016918

Bellan, P. M. (2016). Revised single-spacecraft method for determining wave vector k and resolving space-time ambiguity. Journal of Geophysical Research: Space Physics, 121(9), 8589-8599. https://doi.org/10.1002/2016ja022827

Burch, J. L., Moore, T. E., Torbert, R. B., \& Giles, B. L. (2015). Magnetospheric multiscale overview and science objectives. Space Science Reviews, 199(1-4), 5-21. https://doi.org/10.1007/s11214-015-0164-9

Cassak, P. A., \& Shay, M. A. (2007). Scaling of asymmetric magnetic reconnection: General theory and collisional simulations. Physics of Plasmas, 14(10). https://doi.org/10.1063/1.2795630

Denton, R. E., Anderson, B. J., Ho, G., \& Hamilton, D. C. (1996). Effects of wave superposition on the polarization of electromagnetic ion cyclotron waves. Journal of Geophysical Research, 101(A11), 24869-24885. https://doi.org/10.1029/96ja02251

Dunlop, M., Southwood, D., Glassmeier, K.-H., \& Neubauer, F. (1988). Analysis of multipoint magnetometer data. Advances in Space Research, 8(9-10), 273-277. https://doi.org/10.1016/0273-1177(88)90141-x 
Engebretson, M. J., Posch, J. L., Wygant, J. R., Kletzing, C. A., Lessard, M. R., Huang, C.-L., et al. (2015). Van allen probes, noaa, goes, and ground observations of an intense emic wave event extending over $12 \mathrm{~h}$ in magnetic local time. Journal of Geophysical Research: Space Physics, 120(7), 5465-5488. https://doi.org/10.1002/2015ja021227

Ergun, R. E., Tucker, S., Westfall, J., Goodrich, K. A., Malaspina, D. M., Summers, D., et al. (2014). The axial double probe and fields signal processing for the mms mission. Space Science Reviews, 199(1-4), 167-188. https://doi.org/10.1007/s11214-014-0115-x

Gary, S. P. (1992). The mirror and ion cyclotron anisotropy instabilities. Journal of Geophysical Research, 97(A6), 8519-8529. https://doi. org/10.1029/92ja00299

Gary, S. P., Moldwin, M. B., Thomsen, M. F., Winske, D., \& McComas, D. J. (1994). Hot proton anisotropies and cool proton temperatures in the outer magnetosphere. Journal of Geophysical Research, 99(A12), 23603-23615. https://doi.org/10.1029/94ja02069

Gary, S. P., \& Winske, D. (1990). Computer simulations of electromagnetic instabilities in the plasma sheet boundary layer. Journal of Geophysical Research, 95(A6), 8085-8094. https://doi.org/10.1029/ja095ia06p08085

Gershman, D. J., F-Vinas, A., Dorelli, J. C., Boardsen, S. A., Avanov, L. A., Bellan, P. M., et al. (2017). Wave-particle energy exchange directly observed in a kinetic Alfven-branch wave. Nature Communications, 8, 14719. https://doi.org/10.1038/ncomms14719

Hollweg, J. V. (1999). Kinetic Alfvén wave revisited. Journal of Geophysical Research, 104(A7), 14811-14819. https://doi. org/10.1029/1998ja900132

Hu, Y., \& Denton, R. (2009). Two-dimensional hybrid code simulation of electromagnetic ion cyclotron waves in a dipole magnetic field. Journal of Geophysical Research, 114(A12). https://doi.org/10.1029/2009ja014570

Hu, Y., Denton, R., \& Johnson, J. (2010). Two-dimensional hybrid code simulation of electromagnetic ion cyclotron waves of multi-ion plasmas in a dipole magnetic field. Journal of Geophysical Research, 115(A9). https://doi.org/10.1029/2009ja015158

Kennel, C. F., \& Petschek, H. E. (1966). Limit on stably trapped particle fluxes. Journal of Geophysical Research, 71(1), 1-28. https://doi. org/10.1029/jz071i001p00001

Lee, J. H., Turner, D. L., Toledo-Redondo, S., Vines, S. K., Allen, R. C., Fuselier, S. A., et al. (2019). MMS measurements and modeling of peculiar electromagnetic ion cyclotron waves. Geophysical Research Letters, 46(21), 11622-11631. https://doi.org/10.1029/2019gl085182

Lee, J. H., Turner, D. L., Vines, S. K., Allen, R. C., Toledo-Redondo, S., Bingham, S. T., et al. (2021). Application of cold and hot plasma composition measurements to investigate impacts on dusk-side electromagnetic ion cyclotron waves. Journal of Geophysical Research: Space Physics, 126(1), e2020JA028650. https://doi.org/10.1029/2020ja028650

Lindqvist, P. A., Olsson, G., Torbert, R. B., King, B., Granoff, M., Rau, D., et al. (2014). The spin-plane double probe electric field instrument for mms. Space Science Reviews, 199(1-4), 137-165. https://doi.org/10.1007/s11214-014-0116-9

Li, W. Y., André, M., Khotyaintsev, Y. V., Vaivads, A., Fuselier, S. A., Graham, D. B., et al. (2017). Cold ionospheric ions in the magnetic reconnection outflow region. Journal of Geophysical Research: Space Physics, 122(10), 194-210. https://doi.org/10.1002/2017ja024287

Min, K., Lee, J., Keika, K., \& Li, W. (2012). Global distribution of emic waves derived from Themis observations. Journal of Geophysical Research, 117(A5). https://doi.org/10.1029/2012ja017515

Omidi, N., Thorne, R., \& Bortnik, J. (2011). Hybrid simulations of emic waves in a dipolar magnetic field. Journal of Geophysical Research, 116(A9). https://doi.org/10.1029/2011ja016511

Pinçon, J.-L., \& Glassmeier, K.-H. (2008). Multi-spacecraft methods of wave field characterization. ISSI Scientific Reports Series, 8, 47.

Pollock, C., Moore, T., Jacques, A., Burch, J., Gliese, U., Saito, Y., et al. (2016). Fast plasma investigation for magnetospheric multiscale. Space Science Reviews, 199(1-4), 331-406

Roennmark, K. (1982). Whamp - waves in homogeneous, anisotropic, multicomponent plasmas (Technical Report No. 179), Sweden, Kiruna: Kiruna Geophysical Institute.

Russell, C. T., Anderson, B. J., Baumjohann, W., Bromund, K. R., Dearborn, D., Fischer, D., et al. (2014). The magnetospheric multiscale magnetometers. Space Science Reviews, 199(1-4), 189-256. https://doi.org/10.1007/s11214-014-0057-3

Shi, C., Zhao, J., Huang, C., Wang, T., \& Dunlop, M. W. (2020). Modulation of ionospheric outflow ions by emic waves in the dayside outer magnetosphere. Physics of Plasmas, 27(3). https://doi.org/10.1063/1.5142686

Tanaka, M. (1985). Simulations of heavy ion heating by electromagnetic ion cyclotron waves driven by proton temperature anisotropies. Journal of Geophysical Research, 90(A7). https://doi.org/10.1029/ja090ia07p06459

Thorne, R. M., \& Horne, R. B. (1993). Cyclotron absorption of ion-cyclotron waves at the bi-ion frequency. Geophysical Research Letters, 20(4), 317-320. https://doi.org/10.1029/93gl00089

Toledo-Redondo, S., André, M., Khotyaintsev, Y. V., Lavraud, B., Vaivads, A., Graham, D. B., et al. (2017). Energy budget and mechanisms of cold ion heating in asymmetric magnetic reconnection. Journal of Geophysical Research: Space Physics, 122(9), 9396-9413. https:// doi.org/10.1002/2017ja024553

Toledo-Redondo, S., André, M., Khotyaintsev, Y. V., Vaivads, A., Walsh, A., Li, W., et al. (2016). Cold ion demagnetization near the x-line of magnetic reconnection. Geophysical Research Letters, 43(13), 6759-6767. https://doi.org/10.1002/2016gl069877

Toledo-Redondo, S., Dargent, J., Aunai, N., Lavraud, B., André, M., Li, W., et al. (2018). Perpendicular current reduction caused by cold ions of ionospheric origin in magnetic reconnection at the magnetopause: Particle-in-cell simulations and spacecraft observations. Geophysical Research Letters, 45(19), 10033-10042.

Toledo-Redondo, S., Lavraud, B., Fuselier, S. A., André, M., Khotyaintsev, Y. V., Nakamura, R., et al. (2019). Electrostatic spacecraft potential structure and wake formation effects for characterization of cold ion beams in the earth's magnetosphere. Journal of Geophysical Research: Space Physics, 124(12), 10048-10062.

Toledo-Redondo, S., Vaivads, A., André, M., \& Khotyaintsev, Y. V. (2015). Modification of the Hall physics in magnetic reconnection due to cold ions at the Earth's magnetopause. Geophysical Research Letters, 42(15), 6146-6154. https://doi.org/10.1002/2015gl065129

Trattner, K., Mulcock, J., Petrinec, S., \& Fuselier, S. (2007). Probing the boundary between antiparallel and component reconnection during southward interplanetary magnetic field conditions. Journal of Geophysical Research, 112(A8). https://doi.org/10.1029/2007ja012270

Vines, S. K., Anderson, B. J., Allen, R. C., Denton, R. E., Engebretson, M. J., Johnson, J., et al. (2021). Determining emic wave vector properties through multi-point measurements: The wave curl analysis. Journal of Geophysical Research: Space Physics, e2020JA028922.

Young, D. T., Burch, J. L., Gomez, R. G., De Los Santos, A., Miller, G. P., Wilson, P., et al. (2014). Hot plasma composition analyzer for the magnetospheric multiscale mission. Space Science Reviews, 199(1-4), 407-470. https://doi.org/10.1007/s11214-014-0119-6

Zhang, J. C., Kistler, L. M., Mouikis, C. G., Klecker, B., Sauvaud, J. A., \& Dunlop, M. W. (2011). A statistical study of emic wave-associated $\mathrm{He}^{+}$energization in the outer magnetosphere: Cluster/codif observations. Journal of Geophysical Research, 116(A11). https://doi. org/10.1029/2011ja016690 